

\title{
Microwave magnetic dynamics in highly conducting magnetic nanostructures
}

M. Kostylev, J. Ding, E. Ivanov, S. Samarin, and A. O. Adeyeye

Citation: Journal of Applied Physics 115, 173903 (2014); doi: 10.1063/1.4873897

View online: http://dx.doi.org/10.1063/1.4873897

View Table of Contents: http://scitation.aip.org/content/aip/journal/jap/115/17?ver=pdfcov

Published by the AIP Publishing

\section{Articles you may be interested in}

Microwave magnetic dynamics in ferromagnetic metallic nanostructures lacking inversion symmetry

J. Appl. Phys. 119, 103903 (2016); 10.1063/1.4942828

Impact of conducting nonmagnetic layers on the magnetization dynamics in thin-film magnetic nanostructures J. Appl. Phys. 113, 043927 (2013); 10.1063/1.4789812

Magnetization dynamics and magnetotransport in epitaxial nanostructures

J. Vac. Sci. Technol. A 22, 1371 (2004); 10.1116/1.1692250

High-resolution imaging of fast magnetization dynamics in magnetic nanostructures

Appl. Phys. Lett. 84, 3328 (2004); 10.1063/1.1723698

Dynamical conductivity of EuSe in high magnetic fields

J. Appl. Phys. 50, 1914 (1979); 10.1063/1.327163

The new SR865 2 MHz Lock-In Amplifier ... \$7950

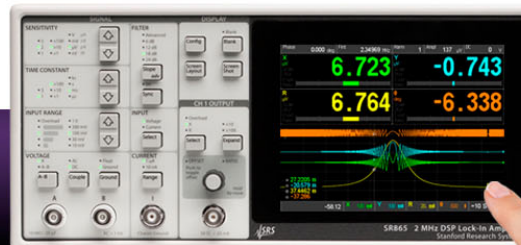

SRS Stanford Research Systems www.thinkSRS.com - Tel: (408)744-9040



Chart recording



(॰)

Intuitive front-panel operation

Touchscreen data display

Save data \& screen shots to USB flash drive

Embedded web server and iOS app

Synch multiple SR865s via $10 \mathrm{MHz}$ timebase I/O

View results on a TV or monitor (HDMl output)

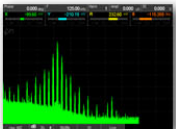

FFT displays

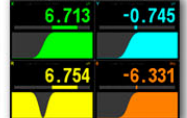

Trend analysis

$1 \mathrm{mHz}$ to $2 \mathrm{MHz}$

$2.5 \mathrm{nV} / \sqrt{ } \mathrm{Hz}$ input noise

$1 \mu$ s to 30 ks time constants

1.25 MHz data streaming rate

Sine out with DC offset

GPIB, RS-232, Ethernet \& USB 


\title{
Microwave magnetic dynamics in highly conducting magnetic nanostructures
}

\author{
M. Kostylev, ${ }^{1}$ J. Ding, ${ }^{2}$ E. Ivanov, ${ }^{1}$ S. Samarin, ${ }^{1}$ and A. O. Adeyeye ${ }^{2}$ \\ ${ }^{1}$ School of Physics M013, The University of Western Australia, Crawley, WA 6009, Australia \\ ${ }^{2}$ Department of Electrical and Computer Engineering, National University of Singapore, Singapore 117576
}

(Received 3 March 2014; accepted 17 April 2014; published online 2 May 2014)

\begin{abstract}
We performed low-noise broadband microstrip ferromagnetic resonance (FMR) measurements of the resonant modes of an array of metallic ferromagnetic nanostripes. In addition to a strong signal of the fundamental mode, we observed up to five weak-amplitude peaks in the field-resolved FMR traces, depending on the frequency. These higher-order absorption peaks have been theoretically identified as due to resonant excitation of odd and even standing spin waves across the direction of confinement in array plane (i.e., across the stripe width). The theory we developed suggests that the odd modes become excited in the spatially uniform microwave field of the FMR setup due to the large conductivity of metals. This promotes excitation of large-amplitude eddy currents in the sample by the incident microwave magnetic field and ultimately results in excitation of these modes. Following this theory, we found that the eddy current contribution is present only for patterned materials and when the microwave magnetic field is incident on one surface of sample surface, as it is in the case of a microstrip FMR. C 2014 AIP Publishing LLC.
\end{abstract}

[http://dx.doi.org/10.1063/1.4873897]

\section{INTRODUCTION}

Ferromagnetic resonance (FMR) is a powerful tool for studying dynamic properties of metallic ferromagnetic thin films and nanostructures (see, e.g., Refs. 1-16). These materials are important for the emerging fields of magnonics ${ }^{17}$ and microwave spintronics. ${ }^{18-20}$

High microwave electric conductivity of metals is important for many spintronic effects, for instance, for the Doppler frequency shift of spin waves ${ }^{21}$ and for spin pumping. ${ }^{22}$ Recently, it has been shown theoretically ${ }^{23}$ and experimentally ${ }^{1}$ that spin pumping may contribute to the resonance linewidth of in-plane standing spin wave (SSW) resonances in in-plane nano-confined geometries.

In addition to the spintronic effects, the conduction currents flowing in a sample result in a trivial effect of microwave Oersted field in it. This may strongly mask the spintronic effects in the experiment, as has been recently shown for the case of the measurements of the Doppler frequency shift for spin waves induced by a dc current flowing in a microscopic stripe made from a ferromagnetic metal. ${ }^{24}$ Thus, understanding of the Oersted-field contribution to the magnetisation dynamics is very important for correct interpretation of experiments involving highly conducting materials.

Furthermore, microwave eddy currents are excited in metallic ferromagnetic films, when the films are exposed to a microwave magnetic field of a ferromagnetic resonance setup. These currents induce a microwave Oersted field, which may lead to the perfect microwave shielding effect under proper experimental conditions, for metallic films with sub-skindepth thicknesses ${ }^{25}$ and result in very strong excitation of higher-order SSW modes across the film thickness. ${ }^{26-31}$

The microstrip or coplanar-line based FMR creates the ideal conditions for observation of the consequences of the perfect microwave shielding. The effect is somewhat similar to the direct injection of microwave currents into the planar sample. ${ }^{32-34}$ In the latter case, a thickness-uniform microwave current across the sample thickness creates a microwave Oersted field whose profile is anti-symmetric in that direction. This field efficiently couples to standing spin wave resonances whose profiles are anti-symmetric across the thickness of the material.

In addition to SSW across the material thickness, ${ }^{10}$ nanopatterned geometries support SSW in the direction of confinement in the sample plane. ${ }^{35}$ In this paper, we take very-lownoise ferromagnetic resonance measurements on a periodic array of $\mathrm{Ni}_{80} \mathrm{Fe}_{20}$ (Permalloy) nanostripes. We observe a number of higher-order absorption peaks which correspond to excitation of both symmetric and anti-symmetric standing spin waves in the sample plane. The amplitudes of the anti-symmetric modes are unexpectedly strong: they are very close to those of the symmetric ones. To explain these amplitudes, we perform calculation which confirms that the non-vanishing amplitudes of these modes are due to excitation of eddy currents in the sample. The theory shows that the amplitudes are non-zero because of the in-plane confinement. The confinement modifies the structure of the eddy-current field and creates a component of the driving microwave magnetic field which efficiently couples to the anti-symmetric resonances.

\section{EXPERIMENT}

A periodic array of permalloy nanostripes of length $4 \mathrm{~mm}$ was fabricated at the National University of Singapore. It was defined in a $240 \mathrm{~nm}$ thick deep ultraviolet lithography resist on top of a $60-\mathrm{nm}$ bottom anti-reflection coating on silicon substrates. To create the stripes, a $25 \mathrm{~nm}$ thick permalloy film was deposited on a substrate having a photoresist pattern on it. ${ }^{36} \mathrm{We}$ used an electron beam deposition system. The base pressure during the deposition was 
$4 \times 10^{-8}$ Torr at the deposition rate was $0.2 \AA /$ s. A lift-off process followed the deposition of the metal. The fabricated stripes have the width $w=300 \mathrm{~nm}$. The edge to-edge separation of the neighboring nano-elements is $d=115 \mathrm{~nm}$. A continuous (unpatterned) reference permalloy film with the same thickness $L=25 \mathrm{~nm}$ was also deposited and used for determining the permalloy magnetic parameters. FMR data taken on it show that its saturation magnetization $4 \pi \mathrm{M}=8700 \mathrm{G}$ and gyromagnetic ratio $\mathrm{g}=2.1$.

Broadband FMR measurements have been taken at the University of Western Australia applying the static magnetic field along the stripe length (i.e., along the $4 \mathrm{~mm}$ size, which is the $y$-direction in Fig. 1). The sample was placed on top of a $0.3 \mathrm{~mm}$-wide microstripe line which runs in the $z$-direction in Fig. 1 and the amplitude of the microwave signal transmitted through the line was measured. (This configuration is shown in Fig. 1 upside down.)

Nano-structuring of magnetic films strongly reduces the amplitude of the FMR response. It becomes comparable with or fall below the noise level. This makes observation of higher-order resonance modes difficult. In order to increase the number of the higher-order modes seen in the FMR absorption spectra on top of the noise level, we used the field modulation method in combination with a high-sensitivity FMR receiver. ${ }^{37}$ In the field-modulation FMR, an extra small-amplitude ac $(220 \mathrm{~Hz})$ magnetic field is applied parallel to the static field and a lock-in amplifier is used to detect the rectified signal from the output of the FMR setup. The lock-in is locked to the field modulation frequency. It enables synchronous demodulation of the signal from the setup output.

In this case, the registered FMR signal takes the form of the first derivative of the Lorentzian curve with respect to the parameter which is swept to register the absorption traces ("differential absorption"). We take measurements appliedfield resolved, when the applied magnetic field $H_{a}$ is swept while keeping the frequency of the microwave signal applied to the sample constant. Sweeping the applied field strongly reduces the number of artifacts seen in the raw FMR absorption spectra.

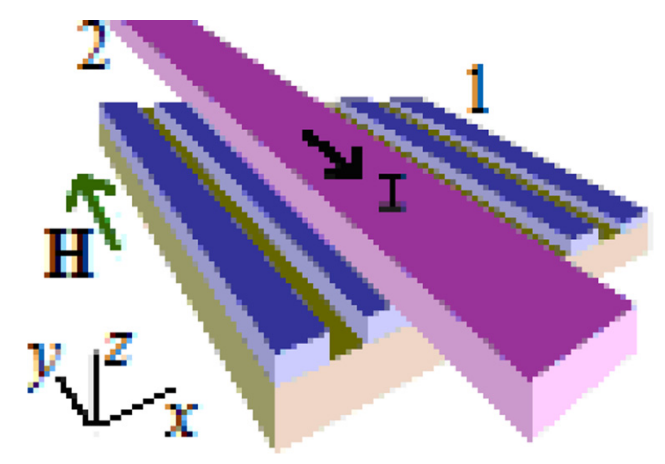

FIG. 1. Sketch of the measurement configuration. 1 is the experimental sample. It has the form of an array of nanostripes formed on a Silicon substrate. 2 is the microstrip line used to take the FMR spectra. $\mathbf{H}$ denotes the static magnetic field applied along the stripes. Note that the nanostripes are shown not to the scale; their actual width is $300 \mathrm{~nm}$ and the width of the microstrip line is $0.3 \mathrm{~mm}$. For the convenience of the theoretical treatment in Sec. III, the configuration is shown upside down with respect to the actual experimental configuration (the sample actually sits on top of the microstrip line).
On top of all these measures, we employed a highsensitivity custom-designed microwave receiver. The noise level of the receiver is at least two orders of magnitude smaller than in the standard approach of direct detection of the output signal of the microstrip line with a diode. ${ }^{3,10}$ This makes a significant number of higher-order modes of standing spin waves across the stripe width easily observable even for such a small stripe thickness.

The results of our measurements are shown in Fig. 2 for a number of frequencies. The first higher-order mode emerges from the zero field above $10 \mathrm{GHz}$, therefore the


Applied field (Oe)

FIG. 2. The experimentally measured FMR absorption traces. The figures in the traces indicate the frequency at which the data were taken. Solid lines: measurements taken with the standard sensitivity. Dashed lines: highsensitivity low-noise data. The vertical arrows indicate the modes which are identified as odd (anti-symmetric) standing waves across the stripe width. 
traces taken below $11 \mathrm{GHz}$ are not shown. The traces taken with the standard sensitivity are shown by the solid lines. One observes a strong fundamental mode (the highest frequency mode) and a number of very small additional peaks at lower applied fields. The low-noise receiver allows us to also take measurements at a much higher sensitivity level of the lock-in amplifier. These data are shown by the dashed line in the figure. The signal of the fundamental mode is clipped in this case, but up to five higher-order absorption peaks become easily resolved. The main observation from this figure is that all these higher-order modes have comparable amplitudes.

\section{THEORY}

\section{A. Resonance peak identification}

To proceed further, we need to identify the resonance modes which give rise to the observed absorption peaks. We carry out identification of these modes by performing numerical modeling of resonance fields for this geometry. We use magnetic parameters extracted from the FMR measurements on the reference continuous film. The same original twodimensional model implemented as a MathCAD worksheet as previously employed in Ref. 34 was utilized to conduct these simulations.

Very briefly, the linearised Landau-Lifshitz equation is transformed into a integro-differential operator defined on the cross-section of an individual stripe. The differential part originates from the effective exchange field and the integral part originates from the contribution of the dynamic dipole field of precessing magnetization vector to the total effective dynamic magnetic field. The dynamic dipole field includes self-fields of individual stripes and dipole coupling between individual stripes. (FMR corresponds to the collective magnonic mode with a zero Bloch wave vector. ${ }^{38}$ )

The values of the resonant fields represent eigenvalues of the operator. Discretization of the operator on a rectangular 2D mesh (width times thickness of the stripe) results in a matrix $C$ whose eigenvalues represent the resonant fields $H_{\mathrm{n}}$. Since in our experiment the sample is in the magnetically saturated state, from the whole set of eigenvalues only a small number of positive values are important. These positive eigenvalues correspond to positive resonance fields, i.e., the fields co-aligned to the static magnetization vector. The eigenvalues of the matrix are calculated by using the QR-algorithm built-in into MathCAD software. The mesh step is $5 \mathrm{~nm}$ in both in-plane and out-of-plane directions. The simulations are quite fast: it takes 1-2 min to complete a simulation run.

The results of the simulations are in good agreement with the experiment in terms of the number of modes existing in the experimental field range for each particular frequency. In particular, similar to the experimental data in Fig. 2 , for $16 \mathrm{GHz}$ the modeling shows that 6 discrete resonances exist in the positive applied field.

The model also allows us to calculate the mode profiles across the stripe cross-section. The profiles represent vector eigen-functions $\left|m_{x}^{n}(x, z), m_{z}^{n}(x, z)\right\rangle$ of the integro-differential operator, where $\mathbf{m}(x, y)$ is the amplitude of dynamic magnetization at the point $(x, y)$ on the nanostripe cross-section, and the respective frame of reference is shown in Fig. 1. In the discrete form, the operator eigen-functions transform into eigenvectors of $C$. They are calculated using a numerical procedure built-in in MathCAD software.

All the modes for the positive fields seen in the calculation demonstrate almost uniform precession across the stripe thickness. Therefore for the convenience of displaying these profiles, we take the mean values of $\left|m_{x}^{n}(x, z), m_{z}^{n}(x, z)\right\rangle$ along $z$. These 1D profiles are shown in Fig. 3. One sees that the
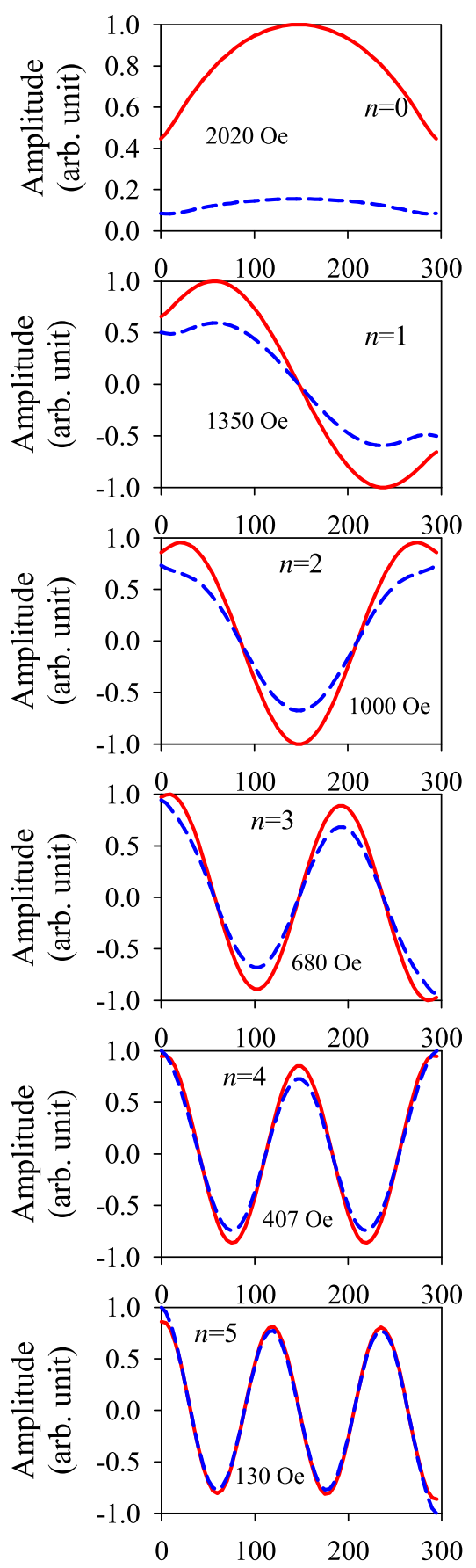

Co-ordinate along stripe width $x(\mathrm{~nm})$

FIG. 3. Calculated mode profiles. The figures in the panels are the mode numbers and the respective calculated resonance fields. Solid lines: the inplane component of dynamic magnetisation $m_{\mathrm{x}}$. Dashed lines: the out-ofplane component $m_{\mathrm{z}}$. Frequency is $15.004 \mathrm{GHz}$. 
fundamental mode $(n=0)$ and two higher-order modes $(n=2)$ and $(n=4)$ have symmetric profiles and the profiles for modes $n=1,3,5$ are anti-symmetric. (The slight asymmetry seen for $n=5$ is because for convenience we plot either real or imaginary part (whichever is much larger) of the complex mode profile for each vector component. The absolute values of $m_{\mathrm{x}}$ and $m_{\mathrm{z}}$ are perfectly symmetric or anti-symmetric.)

As shown in Ref. 10, the amplitudes of the modes $\tilde{\mathbf{m}}^{n}(x, z)$ driven by an external microwave field $\mathbf{h}(x, z)=\left|h_{\mathrm{x}}(x, z), h_{\mathrm{z}}(x, z)\right\rangle$ can be obtained based on the operator eigen-functions

$$
\begin{aligned}
\tilde{\mathbf{m}}^{n}(x, z)= & \left|m_{x}^{n}(x, z), m_{z}^{n}(x, z)\right\rangle \\
& \times \frac{\left\langle m_{x}^{n}(x, z), m_{z}^{n}(x, z) \mid h_{x}(x, z), h_{z}(x, z)\right\rangle}{H_{a}-H_{n}+i \Delta H} .
\end{aligned}
$$

In this expression, $\Delta H$ is the field-resolved resonance linewidth, $\left\langle m_{x}^{n}(x, z), m_{z}^{n}(x, z)\right|$ represents the respective vector eigen-function of the conjugated operator. The object $\langle\ldots \mid \ldots\rangle$ denotes a scalar products of two vectors integrated over the area of the stripe cross-section.

We need the eigen-functions of the conjugated problem, since the operator is not Hermitian. Therefore, its eigenfunctions are not orthogonal to each other, but they are orthogonal to ones of the conjugated operator. (The non-Hermitian character of the operator reflects the presence of the crossproduct in the linearized Landau-Lifshitz equation.) In the discrete formulation of the problem, there is no need to construct the conjugated operator and then calculate its eigen-functions. Numerically its eigen-functions are obtained as respective rows of the matrix which is inverse to the one having $\left|m_{x}^{n}(x, z), m_{z}^{n}(x, z)\right\rangle$ as its columns. This reflects the fact that

$$
\left\langle m_{x}^{l}(x, z), m_{z}^{l}(x, z) \mid m_{x}^{n}(x, z), m_{z}^{n}(x, z)\right\rangle=\delta_{n, l},
$$

where $\delta_{n, l}$ is Kronecker delta.

As shown in Ref. 39, the transmission coefficient of a microstrip loaded by a sample scales as scalar magnetic susceptibility $\kappa$. Furthermore, the contribution to the energy $E_{\mathrm{h}}$ of the spin system due to its coupling to an external microwave field is

$$
E_{h}=\int_{S} \sum_{n} \mathbf{h}(x, z) \tilde{\mathbf{m}}^{n}(x, z) d s,
$$

where $S$ is the area of the strip cross-section, and the sum is taken over the set of the modes exiting in the positive applied field. This allows us to define $\kappa$ as

$$
\kappa=E_{h} /\left(S h_{m s}^{2}\right)
$$

where $h_{m s}$ is the amplitude of the magnetic field of the microwave current in the microstrip line. Here, we used the idea that given the vanishingly small width of an individual magnetic stripe with respect to the width of the microstrip, $\mathbf{h}_{m s}$ can be assumed to be spatially uniform and directed perfectly along $x$.
For insulating continuous magnetic films $\mathbf{h}(x, z)=\mathbf{h}_{m s}$ and $\kappa(4)$ reduces to the mean value $\left\langle\tilde{m}_{x}^{n}\right\rangle$ of $\sum_{n} \tilde{m}_{x}^{n}(x, z)$ over the stripe cross-section

$$
\kappa=\left\langle\tilde{m}_{x}^{n}\right\rangle=\frac{1}{S} \int_{S} \sum_{n} \tilde{\mathbf{m}}^{n}(x, z) d s .
$$

For continuous films with high electric conductivity of metals this field combines with the Oersted field of eddy currents induced by $\mathbf{h}_{m s}$ in the film. The Oersted field is perfectly anti- symmetric across the film thickness and its amplitude at film surfaces equals to $h_{m s}$. As a result, at the surface facing the microstrip line the total microwave magnetic field which drives magnetization precession is $2 h_{m s}$. It drops linearly with the distance from the surface such that at the film surface facing away from the microstrip the driving field vanishes. This perfect microwave shielding promotes efficient excitation of anti-symmetric standing wave modes across the film thickness. ${ }^{25}$

From the symmetry reason, it is clear that the antisymmetric modes are driven by the anti-symmetric eddy-current field component of the total driving field and the symmetric ones by the spatially uniform component $\mathbf{h}_{m s}$. For a $25 \mathrm{~nm}$-thick continuous permalloy film, the first (anti-symmetric) standing spin wave mode across the film thickness does not exist in a positive applied field for our frequency range. Obviously, the same should be valid for nanostripes as well. This is consistent with our simulations of the modal profiles in Fig. 3, as stated above all the profiles are quasi-uniform in the $z$-direction. This implies that one can use the same formula $\mathbf{h}(x, z)=\mathbf{h}_{m s}$ and thus (5) for calculation of the FMR response of the nanopatterned sample. Indeed, the validity of this approach has been proven by the good agreement with the experiment in Ref. 10 .

The result of this calculation is shown in Fig. 4 (dotted line in Panel (b)) in comparison with the experimental FMR

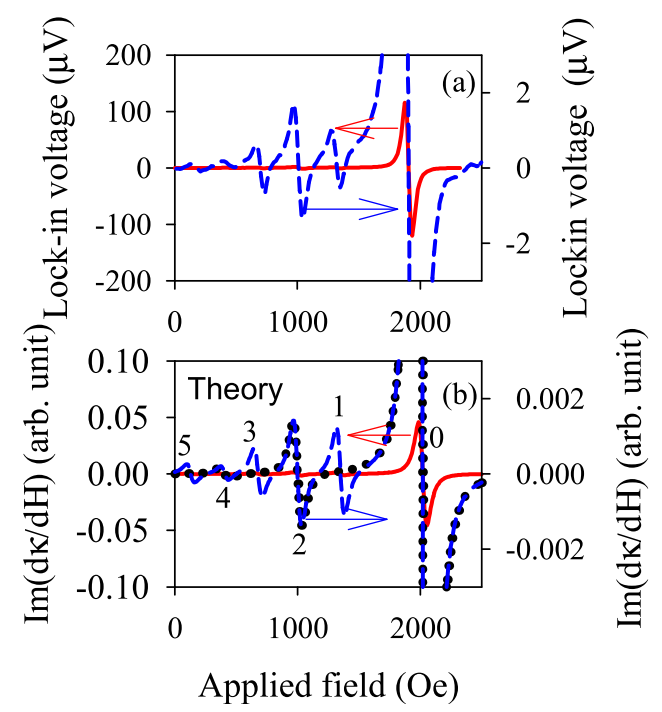

FIG. 4. Experimental (a) and theoretical (b) differential absorption traces for 15.004 GHz. Solid lines: standard sensitivity/un-zoomed; dashed lines: increased sensitivity/zoomed-in. Dotted line in (b): calculation with Eq. (5) which does not take into account the contribution of eddy currents to magnetization dynamics. Numbers in (b): mode numbers for modes in Fig. 3 giving rise to the respective differential absorption peaks. 
trace (Panel (a)). In Fig. 4(b), we plot $\mathrm{d} \kappa / \mathrm{d} H_{\mathrm{a}}$ in accordance with the differential absorption registered in the field-modulated FMR. One sees good agreement of positions and amplitudes of the simulated absorption peaks for $n=0$, 2,4 with experiments. The peaks corresponding to the modes $n=1,3,5$ (Fig. 3) are completely missing in the simulated trace.

This is in agreement with the anti-symmetric character of the latter modes. However, this result is in disagreement with the experiment (Fig. 4(a)) where absorption peaks are also seen at the field positions consistent with the positions of the theoretical anti-symmetric modes.

\section{B. Eddy current contribution to FMR absorption}

In this sub-section, we consider the additional peaks which are seen in the experiment but not seen in the calculation using Eq. (5) (Fig. 4). Based on the simulated resonance fields they are unambiguously identified as the antisymmetric modes $n=1,3,5$ (Fig. 3). We claim that they appear in the absorption spectrum due to contribution from eddy currents in the sample to the microwave driving field. In the following, we support this claim by a simple theoretical model.

The situation is similar to the case of the efficient excitation of anti-symmetric standing spin wave modes across the thickness of a continuous film in a stripline-based FMR ${ }^{16,28}$ which we shortly discussed after Eq. (5) in Sec. III A. It is instructive to give more details of the electrodynamics for the continuous films here before we proceed to the analysis of the nano-patterned materials. The process described after Eq. (5) can be separated in two stages. (i) The spatially uniform microwave magnetic field of the stripline $\mathbf{h}_{m s}$ induces a microwave electric field $\left(e_{\mathrm{y}}\right.$ in the frame of reference of the present paper) in the plane of a continuous metallic film with conductivity $\sigma$. The electric field is generated through electromagnetic induction. Because the film thickness is smaller than the skin depth at microwave frequencies this electric field is uniform across the film thickness. (ii) The microwave current $\sigma e_{\mathrm{y}}$ driven by this field induces an Oersted field $\mathbf{h}^{O e}$ inside the sample. This field is parallel to $\mathbf{h}_{m s}$ and is anti-symmetric across the film thickness as we have already mentioned in Sec. III A. The latter follows just from considering the magnetic field circulation law for the thicknessuniform current density $\sigma e_{\mathrm{y}}$.

The amplitude of $e_{\mathrm{y}}$ for a non-magnetic film with a sub-skin depth thickness is obtained from a solution of the system of Maxwell equations with appropriate boundary conditions (see Appendix 2 of Ref. 25). For the magnetic films, one has to solve self-consistently this system of equations together with the linearized Landau-Lifshitz-Gilbert equation. However, given the very weak microwave absorption by the thin metallic films, the field structure resulting from this self-consistent solution is practically the same as for the non-magnetic films. Therefore, in the following we will use the microwave field structure of a non-magnetic sample with the same geometry and the same value of conductivity to analyze the magnetization dynamics driven by the eddy currents in the ferromagnetic sample.
It is not necessary to evaluate $e_{\mathrm{y}}$ in order to calculate $\mathbf{h}^{O e}$. Because of the effect of the perfect microwave shielding the amplitude of $\mathbf{h}^{O e}$ should be equal to $\mathbf{h}_{m s}$ at the film surface facing the microstrip and to $-\mathbf{h}_{m s}$ at the opposite surface. ${ }^{25}$ Therefore, from the magnetic field circulation law we have

$$
e_{\mathrm{y}}=2 h_{m s} /(\sigma L)
$$

(recall that $L$ is the sample thickness). This result is valid for continuous films.

Let us now consider nanostripes. The aspect ratio $L / w$ for our stripe array is small $(L / w=0.083)$. The stripe edge-toedge separation is also relatively small $d / w=0.38$. Therefore, the microwave field structure should not be very different from the one for a continuous film with the same $L$. This idea allows us to assume that $e_{\mathrm{y}}$ is uniform over the cross-section of the stripe and is given by the same formula (6).

If so, we may now calculate the structure of the Oersted field of the eddy current $\sigma e_{\mathrm{y}}$ by using Biot-Savart Law. This calculation shows that due to the confined geometry, $\mathbf{h}^{O e}$ has now two components: in plane of the sample $h_{x}^{O e}$ and perpendicular to it $h_{z}^{O e}\left(\mathbf{h}^{O e}=\left|h_{x}^{O e}, h_{z}^{O e}\right\rangle\right)$. From the magnetic field circulation law one finds that $h_{x}^{O e}$ should be anti-symmetric across the stripe thickness and quasi-uniform across its width $w$. Therefore, this field component cannot contribute to the excitation of the family of modes of quasi-uniform precession across the film thickness (Fig. 3). For this reason, we would not consider this component in the following.

From the same law, it follows that $h_{z}^{O e}$ should be quasiuniform across the stripe thickness and anti-symmetric across its width. This is confirmed by the calculation based on the Biot-Savart law. For simplicity, it is convenient to consider the value of $h_{z}^{O e}(x, z)$ averaged over the stripe thickness: $\widehat{h}_{z}^{O e}(x)$. This value is given by the following formula:

$$
\widehat{h}_{z}^{O e}(x)=\frac{h_{m s}}{\pi} \sum_{i=-\infty}^{\infty} g(x-i(w+d)) .
$$

The cumbersome expression for $g(x)$ is presented in the Appendix. The sum in (7) takes into account that $\widehat{h}_{z}^{O e}$ is the sum of the self-field generated by the eddy current in each particular stripe and of the Oersted fields of the eddy currents flowing through the neighboring stripes. Accounting to this collective character of the Oersted field noticeably reduces the peak values of the field as follows from Fig. 5. The antisymmetric character of this field is clearly seen from the figure.

We now assume that $\mathbf{h}(x, z)=\left|h_{\mathrm{x}}(x, z), h_{\mathrm{z}}(x, z)\right\rangle$ in Eq. (1) has the form

$$
\mathbf{h}(x, z)=\left|h_{m s}, \widehat{h}_{z}^{O e}(x)\right\rangle .
$$

Substitution of (7) into (1) and (3) results in the trace for $\kappa$ (Eq. (5)) shown in Fig. 4(b) by the dashed line. One sees very good agreement between the theory and the experiment. This confirms that the additional peaks seen in the experiment are the anti-symmetric modes $n=1,3,5$ and that they are excited due to the contribution of the eddy current to the microwave driving field. 


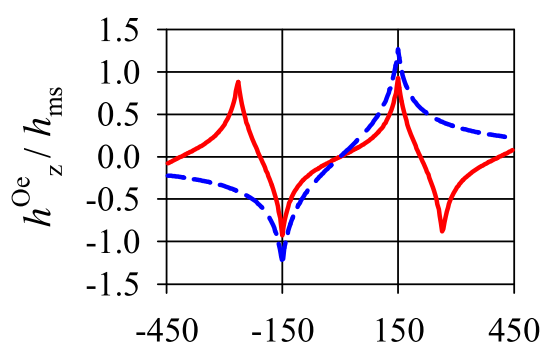

Co-ordinate in the sample plane $x(\mathrm{~nm})$

FIG. 5. The out-of-plane component of the microwave magnetic field of eddy currents induced inside the stripes. Solid line: calculation by using the whole sum in Eq. (7). Dashed line: only the self-field of the stripes is included (the term $i=0$ in the sum in Eq. (7)). The stripe edges are located at $\pm 150 \mathrm{~nm}$. The sharp peaks of the solid line at $\pm 265 \mathrm{~nm}$ correspond to the nearest edges of the neighbouring stripes.

Note that in both experiment and simulation, the amplitude of the mode $n=1$ is smaller that that of $n=2$ mode. Interestingly, if we neglect the collective character of the Oersted field and substitute the Oersted self-field (dashed line in Fig. 5) into Eq. (8) instead of the full Oersted field (solid line in Fig. 5) the amplitude of the $n=1$ peak becomes larger than one of the $n=2$ peak. This demonstrates that the amplitudes (from the experimental results) for the odd modes are driven by the collective Oersted field of the array. As the profile for the collective field is flatter and its peak amplitudes are smaller, the resulting amplitudes of the odd resonance modes reduce when the collective character of the field is "switched on."

Similar simulations were conducted for the other experimental frequencies. The results are in a good agreement with the experimental data (not shown). The FMR responses of the odd modes identified are indicated by the vertical arrows in Fig. 2.

It is noted that although the obtained formulas do not explicitly include the position of the sample with respect to the source of the microwave field, the form of the field source determines the presence of the eddy current contribution to the FMR amplitudes. Indeed, a non-vanishing electric field $e_{\mathrm{y}}$ is induced in a continuous film when the microwave magnetic field $\mathbf{h}_{m s}$ is incident on one film surface only. This is the case of the stripline FMR. In the case of the cavity FMR, the microwave magnetic field is incident on both film surfaces. The electric fields induced by the two magnetic fields incident from the opposite film surfaces are in anti-phase and thus cancel each other. As a result, there is no net Oersted field induced in the sample and the sample behaves as an insulating object (compare Figs. 6(a) and 6(b) in Ref. 28). For this reason, the odd modes $n=1,3,5$ should not be seen when the FMR measurements are taken with a cavity. Unfortunately, since the higher-order modes appear in a positive field first at the frequencies above $10 \mathrm{GHz}$, the standard cavity FMR setup operating at $9.5 \mathrm{GHz}$ cannot be used for checking this idea. One needs a cavity which is able to operate above $13 \mathrm{GHz}$. However, such a cavity is not available in our laboratory.

The only question which still needs to be discussed is why the out-of-plane component $h_{\mathrm{z}}$ of the driving field gives such a strong contribution to the FMR response? For continuous metallic ferromagnetic films it is known that due to the strong ellipticity of precession $\left(\left|m_{z} / m_{x}\right| \ll 1\right)$ the contribution of the $h_{\mathrm{z}}$-component to the FMR response is negligible with respect to the one of the in-plane component $h_{\mathrm{x}}$. A priori one may expect the same for nanopatterned materials. However, the latter statement contradicts our theory.

Indeed, from Fig. 3 one sees that although the ellipticity is very large for $n=0$ mode, it strongly drops for the higherorder modes. This happens due to the qualitatively different structure of the dipole field for these modes with respect to the fundamental one: $n=0$ has no nodes in the profile, but all the other modes have them. The other contribution to the reduction in the ellipticity is the strong exchange contribution to the effective field for $n>0$ modes. The small ellipticity of precession makes it possible to efficiently drive these modes by the out-of-plane component $\widehat{h}_{z}^{O e}$ of the total driving field.

\section{CONCLUSION}

In this work, we performed high-resolution broadband microstrip FMR measurements of the resonant modes of an array of metallic ferromagnetic nanostripes. The low-noise microwave receiver we employed, enabled us to observe up to five additional weak higher-order peaks in the FMR absorption spectra, apart from the strong signal of the fundamental mode. These higher-order modes have been theoretically identified as odd and even standing spin waves across the direction of confinement in the sample plane (i.e., across the stripe width).

The symmetry consideration tells one, however, that the odd modes should not be excited in the spatially uniform microwave magnetic field of a wide microstripe line used to take these measurements. We have developed a theory which shows that the odd modes become excited because the samples have large conductivity of metals. This promotes excitation of a large-amplitude eddy current in the sample by the incident microwave magnetic field. The induced eddy current due to its type of symmetry efficiently couples to the anti-symmetric modes. As a result, these modes become driven and detected in the FMR experiment.

Following this theory, we found that the eddy current contribution is present only when the microwave magnetic field is incident on one sample surface only. This is the case of a stripline FMR. In the case of a cavity FMR, the microwave field is incident on both sample surfaces. The doubleside incidence should completely suppress generation of the eddy currents and make excitation of the odd modes impossible. Experimental check of the latter theoretical idea requires a cavity operational at higher-than-usual frequencies and is beyond the scope of the current work.

The efficient excitation of both odd and even higherorder modes is also facilitated by a drastic decrease in the magnetization precession ellipticity with an increase in the mode number, as our theory demonstrates.

\section{ACKNOWLEDGMENTS}

Financial support by the Australian Research Council and Ministry of Education, Singapore is acknowledged. 


\section{APPENDIX: EXPRESSION ENTERING EQ. (7)}

Here, we give the expression for the quantity $g(x)$ which enters Eq. (7). This expression reads

$$
g=g_{1}+g_{2}+g_{3}+g_{4},
$$

where

$$
\begin{gathered}
g_{1}=L(2 x+w) \operatorname{atan}\left(\frac{2 L}{w+2 x}\right)+L(2 x-w) \operatorname{atan}\left(\frac{2 L}{w-2 x}\right), \\
g_{2}=\frac{L^{2}-x^{2}-w^{2} / 4}{2} \ln \frac{(2 x+w)^{2}+4 L^{2}}{(2 x-w)^{2}+4 L^{2}}, \\
g_{3}=\frac{x^{2}+w^{2} / 4}{2} \ln \frac{(2 x+w)^{2}}{(2 x-w)^{2}},
\end{gathered}
$$

and

$$
g_{4}=\frac{x w}{2} \ln \frac{(2 x+w)^{2}(2 x-w)^{2}}{\left[(2 x-w)^{2}+4 L^{2}\right]\left[(2 x+w)^{2}+4 L^{2}\right]} .
$$

${ }^{1}$ H. T. Nembach, J. M. Shaw, C. T. Boone, and T. J. Silva, Phys. Rev. Lett. 110, 117201 (2013).

${ }^{2}$ B. Heinrich, C. Burrowes, E. Montoya, B. Kardasz, E. Girt, Y. Y. Song, Y. Y. Sun, and M. Z. Wu, Phys. Rev. Lett. 107, 066604 (2011).

${ }^{3}$ M. Belmeguenai, S. Mercone, C. Adamo, L. Mechin, C. Fur, P. Monod, P. Moch, and D. G. Schlom, Phys. Rev. B 81, 054410 (2010).

${ }^{4}$ J. Topp, D. Heitmann, and D. Grundler, Phys. Rev. B 80, 174421 (2009).

${ }^{5}$ G. N. Kakazei, G. R. Aranda, S. A. Bunyaev, V. O. Golub, E. V. Tartakovskaya, A. V. Chumak, A. A. Serga, B. Hillebrands, and K. Y. Guslienko, Phys. Rev. B 86, 054419 (2012).

${ }^{6}$ V. Castel, J. Ben Youssef, F. Boust, R. Weil, B. Pigeau, G. de Loubens, V. V. Naletov, O. Klein, and N. Vukadinovic, Phys. Rev. B 85, 184419 (2012).

${ }^{7}$ G. A. Melkov, Y. Kobljanskyj, V. Novosad, A. N. Slavin, and K. Y. Guslienko, Phys. Rev. B 88, 220407 (2013).

${ }^{8}$ F. G. Aliev, J. F. Sierra, A. A. Awad, G. N. Kakazei, D. S. Han, S. K. Kim, V. Metlushko, B. Ilic, and K. Y. Guslienko, Phys. Rev. B 79, 174433 (2009).

${ }^{9}$ G. Shimon, A. O. Adeyeye, and C. A. Ross, Phys. Rev. B 87, 214422 (2013).

${ }^{10}$ R. Bali, M. Kostylev, D. Tripathy, A. O. Adeyeye, and S. Samarin, Phys. Rev. B 85, 104414 (2012).

${ }^{11}$ N. Ross, M. Kostylev, and R. L. Stamps, J. Appl. Phys. 109, 013906 (2011).
${ }^{12}$ C. S. Chang, M. Kostylev, and E. Ivanov, Appl. Phys. Lett. 102, 142405 (2013).

${ }^{13}$ C. T. Boone, H. T. Nembach, J. M. Shaw, and T. J. Silva, J. Appl. Phys. 113, 153906 (2013).

${ }^{14}$ S. S. Kalarickal, P. Krivosik, M. Z. Wu, C. E. Patton, M. L. Schneider, P. Kabos, T. J. Silva, and J. P. Nibarger, J. Appl. Phys. 99, 093909 (2006).

${ }^{15}$ G. Counil, J. V. Kim, T. Devolder, C. Chappert, K. Shigeto, and Y. Otani, J. Appl. Phys. 95, 5646 (2004).

${ }^{16}$ M. Kostylev, A. A. Stashkevich, A. O. Adeyeye, C. Shakespeare, N. Kostylev, N. Ross, K. Kennewell, R. Magaraggia, Y. Roussigné, and R. L. Stamps, J. Appl. Phys. 108, 103914 (2010).

${ }^{17}$ V. V. Kruglyak, S. O. Demokritov, and D. Grundler, J. Phys. D: Appl. Phys. 43, 264001 (2010).

${ }^{18}$ C. W. Sandweg, Y. Kajiwara, A. V. Chumak, A. A. Serga, V. I. Vasyuchka, M. B. Jungfleisch, E. Saitoh, and B. Hillebrands, Phys. Rev. Lett. 106, 216601 (2011).

${ }^{19}$ V. E. Demidov, S. Urazhdin, E. R. J. Edwards, and S. O. Demokritov, Appl. Phys. Lett. 99, 172501 (2011).

${ }^{20}$ L. Lu, Y. Y. Sun, M. Jantz, and M. Z. Wu, Phys. Rev. Lett. 108, 257202 (2012).

${ }^{21}$ V. Vlaminck and M. Bailleul, Science 322, 410 (2008).

${ }^{22}$ Y. Tserkovnyak, A. Brataas, G. E. W. Bauer, and B. I. Halperin, Rev. Mod. Phys. 77, 1375 (2005).

${ }^{23}$ Y. Tserkovnyak, E. M. Hankiewicz, and G. Vignale, Phys. Rev. B 79, 094415 (2009).

${ }^{24}$ M. Haidar, M. Bailleul, M. Kostylev, and Y. Lao, Phys. Rev. B 89, 094426 (2014).

${ }^{25}$ M. P. Kostylev, J. Appl. Phys. 106, 043903 (2009).

${ }^{26}$ N. Chan, V. Kambersky, and D. Fraitova, J. Magn. Magn. Mater. 214, 93 (2000).

${ }^{27}$ W. E. Bailey, C. Cheng, R. Knut, O. Karis, S. Auffret, S. Zohar, D. Keavney, P. Warnicke, J.-S. Lee, and D. A. Arena, Nat. Commun. 4, 2025 (2013).

${ }^{28}$ K. J. Kennewell, M. Kostylev, M. Ali, A. A. Stashkevich, R. Magaraggia, D. Greig, B. J. Hickey, and R. L. Stamps, J. Appl. Phys. 108, 073917 (2010).

${ }^{29}$ M. Kostylev, J. Appl. Phys. 112, 093901 (2012).

${ }^{30}$ M. Kostylev, J. Appl. Phys. 113, 053908 (2013).

${ }^{31}$ I. S. Maksymov and M. Kostylev, J. Appl. Phys. 113, 043927 (2013).

${ }^{32} \mathrm{P}$. Wolf, Z. Angew. Phys. 14, 213 (1962).

${ }^{33}$ Y. V. Khivintsev, L. Reisman, J. Lovejoy, R. Adam, C. M. Schneider, R. E. Camley, and Z. J. Celinski, J. Appl. Phys. 108, 023907 (2010).

${ }^{34}$ C. S. Chang, M. Kostylev, A. O. Adeyeye, M. Bailleul, and S. Samarin, Europhys. Lett. 96, 57007 (2011).

${ }^{35}$ C. Mathieu, J. Jorzick, A. Frank, S. O. Demokritov, A. N. Slavin, B. Hillebrands, B. Bartenlian, C. Chappert, D. Decanini, F. Rousseaux, and E. Cambril, Phys. Rev. Lett. 81, 3968 (1998).

${ }^{36}$ G. Gubbiotti, S. Tacchi, M. Madami, G. Carlotti, A. O. Adeyeye, and M. Kostylev, J. Phys. D: Appl. Phys. 43, 264003 (2010).

${ }^{37}$ E. Ivanov and M. Kostylev, e-print arXiv:1402.3459.

${ }^{38}$ M. P. Kostylev, A. A. Stashkevich, and N. A. Sergeeva, Phys. Rev. B 69, 064408 (2004).

${ }^{39}$ Y. Ding, T. J. Klemmer, and T. M. Crawford, J. Appl. Phys. 96, 2969 (2004). 